\section{Insulin signaling and tight glucose control: a genetic link?}

\section{Alessandro Laviano, Maurizio Muscaritoli and Alessio Molfino}

We read with much interest the article by David B. Sacks [Therapy: Intensive glucose control in the ICU: is sugar nice? Nat. Rev. Endocrinol. 5, 473-474 (2009)] which analyses the interesting point of tight glycemic control in the intensive care unit (ICU). ${ }^{1}$ Whether intensive glucose control should be actively pursued in critically ill patients remains a debated issue. In previous trials, tight glycemic control has been demonstrated to reduce mortality, ${ }^{2,3}$ but the NICE-SUGAR study appears to contradict these findings. ${ }^{4} \mathrm{~A}$ number of factors related to the selection criteria, the type of patients enrolled and the intervention schedule may explain the controversial results obtained by the different trials. However, patients' specific genetic profiles may well be involved in contributing to the clinical outcome observed with tight glycemic control. In this light, it could be speculated that although phenotype-associated criteria were rigorously met during patient selection, the patients enrolled in the trials represented a genetically mixed population.
The influence of caloric restriction on tumor growth is an unresolved issue. Some tumors are sensitive to caloric restriction, but cancer cells also exist whose metabolism is not influenced by the availability of nutritional substrates. Explaining at least in part the lack of a consistent response to fasting by tumors, Kalaany and Sabatini ${ }^{5}$ showed that cancer cells forming tumors resistant to dietary restriction carry mutations that determine constitutive activation of the phosphatidylinositol-3-kinase (PI3K) pathway. In addition, these cancer cells proliferate in culture without the addition of insulin. ${ }^{5}$ Similar activating mutations may exist in humans, and considering that PI3K is an essential component of the insulin signaling pathway, it could be proposed that intensive glucose control may yield increased survival only when pursued in those critically ill patients whose insulin signaling is constitutively activated. Interestingly, the controversial results on clinical outcomes of intensive glucose control have been obtained in populations from different geographical areas $^{2-4}$ and thus of possibly different genetic backgrounds. Therefore, the stratification of patients for PI3K mutations, rather than intensive glucose control per se, may be a key factor in reducing mortality in critically ill patients.

Department of Clinical Medicine, Sapienza University, viale dell'Università 37, 00185 Rome, Italy (A. Laviano, M. Muscaritoli, A. Molfino).

Correspondence to: A. Laviano alessandro.laviano@uniroma1.it

doi:10.1038/nrendo.2009.156-c1

Competing interests

The authors declare no competing interests.

1. Sacks, D. B. Therapy: Intensive glucose control in the ICU: is sugar nice? Nat. Rev. Endocrinol. 5, 473-474 (2009).

2. van den Berghe, G. et al. Intensive insulin therapy in the critically ill patients. N. Engl. J. Med. 345, 1359-1367 (2001).

3. van den Berghe, G. et al. Intensive insulin therapy in the medical ICU. N. Engl. J. Med. 354, 449-461 (2006).

4. NICE-SUGAR study investigators. Intensive versus conventional glucose control in critically ill patients. N. Engl. J. Med. 360, 1283-1297 (2009).

5. Kalaany, N. Y. \& Sabatini, D. M. Tumours with $\mathrm{PI} 3 \mathrm{~K}$ activation are resistant to dietary restriction. Nature 458, 725-731 (2009). 